\title{
Parkinson disease in eldery patients: lessons from odour detection thresholds on olfacto-trigeminal interaction*
}

\author{
Clovis Foguem ${ }^{1,2}$, Mohamed Lemdani ${ }^{3}$, Caroline Huart ${ }^{4,5}$ \\ 'Auban Moët Hospital, Epernay, France \\ 2 University of Burgundy, Dijon, France \\ ${ }^{3}$ Faculty of Pharmaceutical and Biological Sciences, EA 2694, University of Lille II, Lille, France \\ ${ }^{4}$ Department of Otorhinolaryngology, Cliniques universitaires Saint-Luc, Brussels, Belgium \\ ${ }^{5}$ Institute of Neuroscience, Université catholique de Louvain, Brussels, Belgium
}

Rhinology 56; 2: 127-132, 2018

https://doi.org/10.4193/Rhin17.016

*Received for publication:

January 21, 2017

Accepted: November 14, 2017

Background: Human nasal chemosensation is mediated by two separate, though interacting sensory pathways: the trigeminal and olfactory systems. Trigeminal sensitivity and olfacto-trigeminal interactions have not yet been well studied in idiopathic Parkinson's disease (IPD).

Objectives: The aim of this study was to assess odour detection thresholds in elderly IPD patients, and compare them to the odour detection thresholds of healthy controls. Finally, we investigated potential interactions between trigeminal and olfactory sensitivity.

Methods: 89 IPD patients aged over 65 and 89 matched healthy participants were enrolled in the study. Odour detection thresholds to 3 stimuli differentially activating olfactory and trigeminal afferents (Phenyl-ethyl alcohol, $\mathrm{n}$-Butanol and Pyridine) were assessed, using an ascending staircase, binary forced-choice procedure.

Results and conclusion: Detection threshold scores were able to discriminate between elderly IPD and controls. Pyridine was less effective than the two other odorants, suggesting that trigeminal pathway is less impaired than the olfactory system. We found that the detection thresholds were significantly different between IPD patients with good autonomy, and patients with impaired autonomy.

Key words: neurodegenerative, odour detection thresholds, olfacto-trigeminal interaction, smell abnormalities, Parkinson's diagnosis

\section{Introduction}

The impairment of olfaction is now increasingly recognized as a neurodegenerative diseases feature ${ }^{(1)}$ and a prominent early-appearing feature of idiopathic Parkinson's disease (IPD) (2); and the American Academy of Neurology even recommends an olfactory evaluation of patients, as part of the basic clinical evaluation $^{(3)}$. Although lots of studies have investigated olfaction in IPD patients, the vast majority of them have mainly focused on relatively young patients ( $<65$ years), while elderly IPD ( $>65$ years) have hardly been investigated.

It is well known that the vast majority of odorants activate both olfactory system (mediated by the first cranial nerve (CN I)) and trigeminal system (mediated by CN V). These two systems closely interact with each other, resulting in the global nasal chemosensory experience ${ }^{(4,5)}$. While the decrease in olfactory sensitivity is well recognized in IPD regardless of age; in contrast, trigeminal sensitivity appears to be preserved in elderly patients with IPD ${ }^{(6)}$. Hence, it can be hypothesized that trigeminal sensitivity is less affected in IPD as compared to pure olfactory sensitivity.

The purposes of the present study were thus: 1) to assess nasal chemosensory perception deficits in elderly patients with IPD relative to matched healthy control participants, on the basis of odorants detection thresholds; 2 ) to investigate a possible interaction between olfactory and trigeminal systems, using odorants that are known to differentially activate the olfatory and 
trigeminal systems; and 3) to explore potential links between chemosensory detection thresholds.

\section{Materials and methods}

The study is analytical, cross-sectional and aims to evaluate the olfactory detection thresholds in Parkinson's disease patients and matched healthy controls. It was conducted according to the Declaration of Helsinki on Biomedical Research Involving Human Subjects.

During enrollment and data collections from October 2011 to May 2014, participants and their relatives were given detailed information about all testing procedures and their consents were collected prior to participating in the study.

\section{Participants}

In total, 178 subjects were enrolled in the present study: 89 elderly patients with Parkinson disease (IPD) aged 65 and over [74.80 \pm 6.76 years; range: 65 - 90 years] and healthy controls matched for gender [79.80 \pm 8.82 years; range: 65 - 93 years]. All participants had no history of nasal/sinus and oral/throat diseases, neither head injury nor stroke within six months prior to the olfactory tests. Furthermore, they had no acute upper respiratory disease at the moment of testing. Participants in both groups both groups either did not have any history of active smoking or had a less than 20 packs/year of tobacco consumption during the past 10 years. Additionally, during the testing period, participants were free of untreated patent depression [evaluated by Mini-Geriatric Depression Scale ${ }^{(7)}$ ]. Neurocognitive [Folstein's Mini-Mental State Examination (MMSE) ${ }^{(8)}$ and clock drawing ${ }^{(9)}$ ] tests were performed to exclude mild cognitive impairment or moderate and severe dementia ${ }^{(10)}$.

\section{Patient Group}

The IPD patients group included 47 women and 42 men, who were diagnosed according to the United Kingdom Parkinson Disease Society Brain Bank (UKPDSBB) diagnostic criteria (11). Elderly IPD patients were defined as older than 70 years ${ }^{(12,13)}$. We defined three groups of patients: 2 groups of patients, older than 70: 1) early onset elderly IPD consisting primarily of patients whose first clinical signs appeared before the age of 70 (12,14), referred to "IPD1's group" throughout the manuscript (45 out to 89 IPD); 2) late onset IPD patients (occurring after 70), referred to "IPD2's group" throughout the manuscript (23 out to 89 IPD). Moreover, we defined as a third group 3) IPD patients aged between 65 and 70 years, referred to "IPD0's group" (21 out to 89 IPD). "Unified Parkinson's Disease Rating Scale" (UPDRS) (part III) ${ }^{(15)}$ and 'Hoehn and Yahr' (HY) scale ${ }^{(16)}$ [somewhat modified into 5 stages: $\mathrm{HY} 1, \mathrm{HY} 2, \mathrm{HY} 3, \mathrm{HY} 4, \mathrm{HY} 5$ ] evaluations were used to assess (i) severity progression of motor (symptom) impairment; and (ii) broad categories of motor function and relative level of disability, correlated to global clinical autonomy of the IPD's patients, respectively.

At the time of olfactory testing, most of the patients were being treated with L-Dopa (85 of 89 IPD) or some other antiparkinsonian drugs either alone or in a combination of two or more treatments [Dopamine receptor agonists or anticholinergic antiparkinson agents or selective monoamine oxidase $B(M A O B)$ inhibitors (45 out of 89 IPD), catechol-O-methyl transferase (COMT) inhibitors (39 out of 89 IPD) and cranial electrostimulation (7 out of 89 IPD)].

\section{Control Group}

None of the control subjects had a medical history of Parkinsonism, or any other major neurological disorder. Otherwise, participants in this group were in a good state of autonomy.

\section{Olfactory testing \\ Stimuli}

Olfactory detection thresholds were determined for three odorants known to differentially activate the olfactory and trigeminal systems based on the previous data ${ }^{(17)}$. Phenyl-ethyl alcohol (PEA) was chosen to activate almost exclusively the olfactory system; n-Butanol was considered to activate both olfactory and trigeminal systems ${ }^{(6)}$. Finally, Pyridine was used to be specifically activating trigeminal afferents ${ }^{(5)}$.

\section{Procedure}

Successive dilutions of odorants by a factor 2 were realized with distilled water as solvent. This yielded geometric series starting from solutions of pure $n$-Butanol until the 20th dilution, pure PEA until the 23rd dilution or pure Pyridine up to the 26th dilution.

The odorant stimulus was presented in a white glass bottle ( $7.5 \mathrm{~cm}$ high, opening diameter: $1 \mathrm{~cm}$ ) filled with $4 \mathrm{ml}$ of liquid. The bottle was presented for 3 seconds, medially $1 \mathrm{~cm}$ under both nostrils using a holder to avoid any olfactory or thermic interference with the experimenter's hand. Odour thresholds were assessed using an ascending staircase, binary (stimulus vs. blank) forced-choice procedure, with inter-trial intervals of 90 seconds. The two bottles were presented to the subject in random order. After sniffing each stimulus, the participant was asked to identify the one who smelled stronger. An incorrect choice led to increase the concentration of the stimulus in the next trial. The dilution step at which the odorant stimulus was first detected correctly three times in a row was recorded as the detection threshold (Table 1).

\section{Data analysis}

Statistical analyses were performed using Statview (SAS Institute Inc., Version 9.2).

The Shapiro-Wilk test was used to test each variable for normality. As some variables were not normally distributed, nonpa- 
Table 1. Example of ascending forced choice test's notation: the recorded detection threshold was first detected correctly three times in a row.

\begin{tabular}{ccccc} 
& Dilution & Test 1 & Test 2 & Test 3 \\
\hline & 4 & 0 & $X$ & $X$ \\
3 & 2 & $X$ & 0 & $X$ \\
& 1 & 0 & 0 & 0 \\
& & 0 & 0 & 0
\end{tabular}

$\mathrm{O}$ : adequate response ; $\mathrm{X}$ : wrong answer

rametric test procedures (with post-hoc tests when necessary) were used to compare odour detection thresholds. We investigated for a possible order effect of odour (PEA and pyridine) presentation's sequence. Multivariate analyses were conducted, to account for other parameters in our data (age, sex and order of presentation of PEA's stimuli).

In IPD patients, Spearman correlation analyses between odour thresholds were performed. In all cases, the alpha level was set at $\mathrm{p}<0.05$.

Sensitivity and specificity of each odorant to discriminate between IPD patients and healthy controls were assessed using Receiver Operating Characteristic (ROC) curves ${ }^{(18)}$. The area under the ROC curve (AUC) was calculated using SPSS software ${ }^{(19)}$. Youden's index (Youden Index= Sensitivity + Specificity - 1) was used to define the optimal cut-off points.

\section{Results}

\section{Statistical analyses}

According to the Mann-Whitney-Wilcoxon test, median olfactory detection thresholds (PEA, n-Butanol, Pyridine) were found to be lower in IPD patients as compared to healthy matched controls (all $p$ value $<0.001$ ) (Table 2).

The multivariate analysis confirmed a significant difference in the mean detection threshold for PEA between IPD patients (PEA thresholds scores $=13.73 \pm 7.69[0 ; 23]$ ) and controls (PEA thresholds scores $=21.84 \pm 2.07$ [9; 23]) $(F=88.711 ; p<$ 0.000 ), even after controlling for PEA and trigeminal-like stimuli (n-Butanol or Pyridine) order presentation effect. Indeed, we found a significant reduction of the detection thresholds scores for PEA if the trigeminal-like stimuli were presented before PEA (PEA thresholds scores $=16.99 \pm 7.30$ ) compared to if PEA is presented before trigeminal-like stimuli (PEA thresholds scores $=18.86 \pm 6.40)(\mathrm{F}=4.919 ; \mathrm{p}=0.028)$.

The multivariate analysis indicated that the 'age' variable had no impact on the value of odour detection thresholds when compared between IPD patients and controls $(p=0.791 ; 0.822$ and 0.207 for PEA, n-Butanol, and Pyridine, respectively). Moreover, the IPD group (IPD0, IPD1, IPD2) had no significant
Table 2. Comparisons of Phenyl-ethyl alcohol (PEA), n-butanol and pyridine detection thresholds between idiopathic Parkinson's disease (IPD)'s patients and healthy controls.

\begin{tabular}{|c|c|c|c|c|}
\hline & $\begin{array}{c}\text { Number of } \\
\text { subjects } \\
\text { (IPD/ } \\
\text { Controls) }\end{array}$ & $\begin{array}{c}\text { Median } \\
\text { (and } \\
25 \% / 75 \% \\
\text { quartiles) } \\
\text { detection } \\
\text { threshold } \\
\text { values }\end{array}$ & $\begin{array}{l}\text { Mean odor } \\
\text { detection } \\
\text { threshold } \\
\pm \text { SD (IPD/ } \\
\text { Controls) }\end{array}$ & p-value \\
\hline PEA & $89 / 89$ & $21(15 / 23)$ & $\begin{array}{l}13.73 \pm 7.69 / \\
21.84 \pm 2.07\end{array}$ & $<0.0001$ \\
\hline n-Butanol & $89 / 89$ & $19(14 / 20)$ & $\begin{array}{c}12.63 \pm 7.25 / \\
19.34 \pm 1.71\end{array}$ & $<0.0001$ \\
\hline Pyridine & $54 / 76$ & $25(14 / 20)$ & $\begin{array}{l}17.22 \pm 9.40 / \\
24.88 \pm 1.67\end{array}$ & 0.0004 \\
\hline
\end{tabular}

SD: standard deviation; $\mathrm{p}$ (Wilcoxon test); value of significance $<0.05$

Table 3. Comparisons of Phenyl-ethyl alcohol (PEA), n-butanol and pyridine detection thresholds between idiopathic Parkinson's disease (IPD)'s patients treated with COMT inhibitor drugs (eg: Entacapone) and those without COMT inhibitor drugs.

\begin{tabular}{|lcccc}
\hline & $\begin{array}{c}\text { Number of } \\
\text { subjects } \\
\text { (with/with- } \\
\text { out COMT } \\
\text { inhibitor } \\
\text { drugs) }\end{array}$ & $\begin{array}{c}\text { Median } \\
\text { odor } \\
\text { detection } \\
\text { threshold } \\
\text { values }\end{array}$ & $\begin{array}{c}\text { Odor } \\
\text { threshold } \\
\text { quartiles } \\
\text { values }\end{array}$ & p-value \\
\hline (25\%/75\%) & \\
\hline PEA & $39 / 47$ & 16.5 & $8 / 20$ & 0.081 \\
\hline n-Butanol & $39 / 47$ & 16 & $6 / 20$ & 0.0255 \\
\hline Pyridine & $24 / 27$ & 23 & $9 / 26$ & 0.513 \\
\hline
\end{tabular}

effect on the mean odour detection thresholds $(p=0.664,0.271$ and 0.486 for PEA, n-Butanol and Pyridine, respectively). Interestingly, we found that odour detection threshold performances were significantly affected by the autonomy status of patients (evaluated on Hoehn and Yahr's scale). Indeed, patients with benign IPD (honeymoon phase corresponding to HY1 and HY2) had significantly better olfactory detection threshold performances as compared to patients with malignant IPD (corresponding to HY3, HY4 and HY5); [p $<0.005$ for odour (PEA, n-Butanol and Pyridine) thresholds)] (Figure 1). On contrast, no significant difference was observed when the 5 different Hoehn and Yahr's stages were considered separately; although the $\mathrm{p}$-values were close to significance between stages 2 and $3(\mathrm{p}=$ $0.065,0.085$ and 0.088 for PEA, n-butanol and pyridine, respectively).

Finally, we found a significant difference for the n-butanol detection threshold between patients treated with COMT inhibitors 

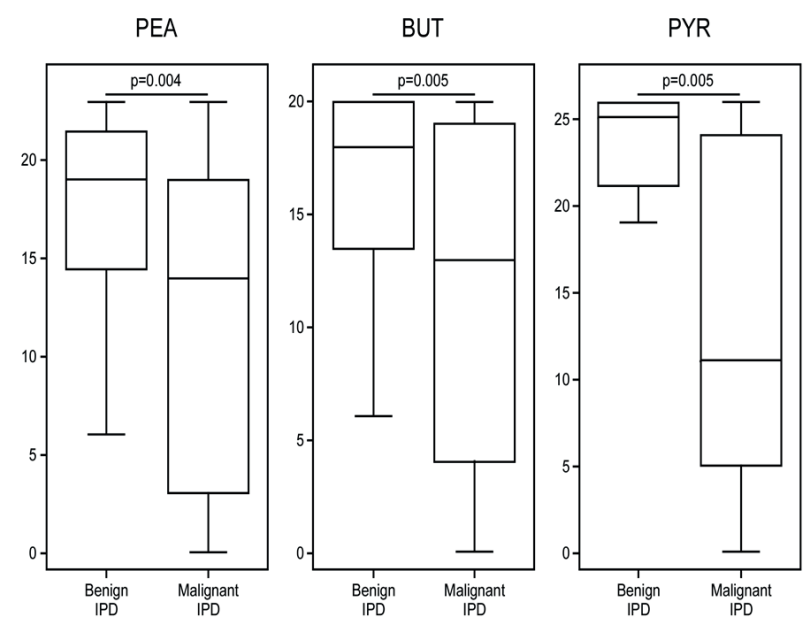

Figure 1. Comparison of odor (PEA, n-Butanol, Pyridine) detection thresholds between benign IPD's patients (during 'honeymoon's phase) and malignant IPD patients.

$\mathrm{p}$ (Wilcoxon test); value of significance < 0.05. PEA: Phenyl-ethyl alcohol; BUT: n-Butanol; PYR: Pyridine .
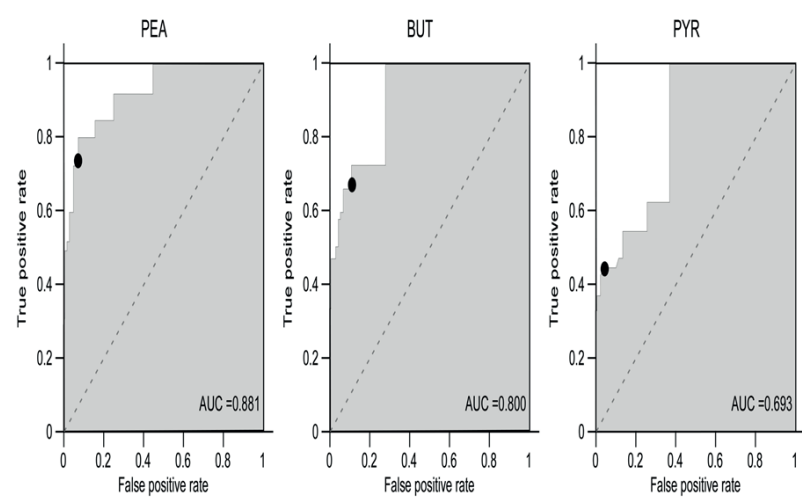

Figure 2. Receiver Operating Characteristic (ROC) curves. ROC curves were computed to estimate the discrimination performance (ability to discriminate between IPD patients and controls) of Phenyl-ethyl alcohol (PEA), n-Butanol (BUT) and Pyridine (PYR). The black dot indicates the optimal cut-off value, as defined by the Youden index. Optimal cut-off values ( 1 -Specificity; Sensitivity) were $(0.08 ; 0.72),(0.11 ; 0.66)$ and $(0.04$; 0.44) for PEA, n-Butanol and Pyridine, respectively. The threshold scores associated with the highest Youden index were 20 for PEA, 19 for BUT and 22 for PYR. AUC: area under the ROC curve. drugs (e.g., Entacapone) associated or not with other drugs and patients without COMT inhibitors drugs (Table 3). This was not true considering thresholds to PEA and Pyridine. L-dopa, dopamine agonists and deep brain stimulation had no effect on odour detection thresholds.

\section{Correlation analysis results}

We found a significant correlation between the different odour detection thresholds performances [PEA- $n-B u t a n o l(p<0.001$, $r=0.78)$; PEA-Pyridine ( $p<0.001, r=0.78)$; and $n$-Butanol-Pyridine $(p<0.001, r=0.81))]$ according to Spearman coefficient.

\section{Receiver operating characteristic (ROC) curves and Odours} threshold's cut-off points

The ability of each odour to discriminate between IPD and controls was assessed using ROC curves. We found that detection threshold scores to n-Butanol and PEA had good discrimination performances, with sensitivity and specificity of respectively $66 \%$ and $89 \%$ for $n$-Butanol (area under the curve $(A \cup C)=$ 0.800), and $72 \%$ and $92 \%$ for PEA (AUC $=0.881$ ). The pyridine's detection thresholds discrimination performance was lower (Sensitivity: 44\%, Specificity: 96\%, AUC = 0.693) (Figure 2). The threshold scores associated with the highest Youden index were 20 for PEA, 19 for $n$-Butanol and 22 for Pyridine (Figure 2).

\section{Discussion}

This study shows that similarly as for younger IPD patients (less than 65 years), elderly IPD patients have impaired olfactory detection when compared to healthy subjects. We observed significantly lower mean odour detection performances to the 3 odorants in IPD patients as compared to healthy elderly controls. Because the odorants used in the present study activate the olfactory and/or trigeminal systems, the results suggest that the overall nasal chemosensory sensitivity is more affected in elderly patients with IPD as compared to healthy controls. It is well known that olfaction decreases with age ${ }^{(20)}$. Similarly, it has been shown that trigeminal sensitivity also decreases with age ${ }^{(21)}$. However, these two senses seem to be even more affected in IPD patients. Hence, it has been proposed that an olfactory test should be systematically used in the clinical workup of IPD patients ${ }^{(1,22)}$. In the present study we found that olfactory thresholds testing using PEA, n-Butanol and Pyridine may be useful in the diagnosis of IPD in the elderly, with IPD patients exhibiting higher odour thresholds as compared to healthy controls. We found that the optimal cutoff value allowing the detection of IPD was 20 (out of 23) for PEA; 19 (out of 20) for $n$ Butanol and 22 (out of 26) for Pyridine.

The ROC curves revealed that pyridine's detection threshold ( $A \cup C=0.693$ ) was less discriminant as compared to $n$-Butanol $(A \cup C=0.800)$ and to PEA $(A \cup C=0.881)$. Having an AUC of less than 0.7 , the discrimination performance of pyridine detection threshold appeared to be poor and not sensitive enough to allow for an adequate discrimination between IPD elderly patients and healthy controls. A possible explanation for this finding is that trigeminal sensitivity could be less impaired in elderly with IPD than olfactory sensitivity as compared to healthy controls. This is in line with a previous study ${ }^{(6)}$. We found highly significant correlations between pairwise 
odour thresholds. These significant correlations between the PEA, $n$-butanol and pyridine detection thresholds could be supported by the existence of a close interaction between the trigeminal and olfactory systems. However, the underlying mechanisms are not yet fully understood ${ }^{(4)}$.

We noticed that a suppression-like interaction mechanism seems to be present between trigeminal and olfactory systems because we found a significant decrease of PEA detection thresholds whenever $\mathrm{n}$-Butanol or Pyridine (with pronounced trigeminal component) were presented to subjects during testing before PEA (having almost no trigeminal effect and mainly olfactory component) $(p=0.028)$. These results are in agreement with those of Schriever et al. ${ }^{(23)}$, who also found a decreased olfactory response due to trigeminal activation. This effect appears to be mediated in the olfactory periphery by neuropeptides such as calcitonin gene-related peptide (CGRP) ${ }^{(23)}$. Importantly, this stimuli order effect was not significantly affecting the results, as revealed by a multivariate analysis.

Moreover, in the IPD patients, we found that patients with benign IPD (honeymoon phase), had significantly better detection thresholds to the three odorants, than patients with malignant IPD showing a loss of autonomy (more gait and balance difficulties, more depression, cognitive problems, swallowing difficulties and autonomic dysfunction). Stern et al. have also shown subtle olfactory test differences between benign IPD and malignant IPD patients using University of Pennsylvania Smell Identification Test (UPSIT) ${ }^{[24]}$, which is an olfactory test based on identification of odour and not on detection thresholds. They found a significantly higher USIPT score in benign IPD patients compared to patients with malignant IPD. However, most of the previous publications found that the olfactory loss appears to be relatively stable over time and is unrelated to the magnitude of IPD motor symptoms (degree of tremor, rigidity, bradykinesia, or gait disturbance) ${ }^{(25)}$. But some of these surveys included early-onset IPD patients, with more or less preserved autonomy or with average Hoehn and Yahr score (disease stage) less than $3^{(25,26)}$. Our findings indicate that even though the olfactory dysfunctions are set up at early PD stage ${ }^{(26,27)}$, the patients may have a sustained slightly decrease of odour (PEA, n-butanol and pyridine) detection thresholds scores with 'inflection point' upon HY2 allowing to discriminate between benign IPD and malignant IPD patients, even if the conventional Hoehn and Yahr's scale does not. Our findings are in agreement with those of Meusel et al., who showed, an overall decreasing olfactory function in 19 PD patients at 5 -year intervals ${ }^{(26)}$. This is likely to be pathology's reflection on olfactory and trigeminal pathways. The severity of the olfactory loss may therefore be used as an indicator of overall disease progression in elderly IPD patients. Nevertheless, it would be interesting to evaluate the progression of olfactory function in parallel with the progression of the disease in future studies. Olfactory dysfunction is a clinically significant problem, with a high burden on quality of life ${ }^{(1)}$, and is likely to grow in prevalence due to demographic shifts and improvement of life expectancy (in general and in IPD patients). For these reasons, we think that olfactory evaluation should be integrated to the clinical follow-up of elderly IPD patients.

Finally, no gender-related differences were apparent for odour detection thresholds comparison ( $p$-values $>0.2$ ); although many studies have shown that women often outperformed men in most subtypes of olfactory tests or examinations ${ }^{(24)}$. One possibility may be related to the advanced age of our population cohort (aged over 65 years) and the fact that olfactory sense sensitivity declines with senescence ${ }^{(28)}$ or some neurodegenerative diseases such IPD. Further studies are warranted to answer this question.

\section{Conclusion}

This study highlights the importance of PEA, n-butanol and pyridine detection thresholds to distinguish between elderly Parkinson's patients and matched healthy controls. Interestingly, we found that odour detection thresholds tests are able to distinguish between the earlier IPD patients with good autonomy (honeymoon phase) and other IPD patients with impaired autonomy (becoming malignant IPD).

Our results suggest that both olfactory and trigeminal systems are impaired in elderly IPD patients, although 'pure' trigeminal pathways seem to be less impaired than the olfactory system.

\section{Acknowledgements}

We would like to thank Dr Gerard Brand with whom this work and the olfactory tests have begun; and Dr Bernard Kamsu Foguem, Dr Donatien Chedom Fotso, Dr Henry Abanda and Dr Ibrahim Mounchetrou Njoya and Mrs Tchuente Foguem Germaine for their contribution and analyses of the data; and $\mathrm{Mr}$ Emanuel Van den Broeke for his careful reading of this manuscript. Finally we are grateful to an anonymous referee whose careful writing and pertinent remarks allowed improving the manuscript's content.

A Health Research Advancement Award of the 'Groupe Pasteur Mutualité' foundation was awarded to Dr Clovis Foguem. Dr Caroline Huart is a postdoctoral fellow supported by the Fund for Scientific Research (FRS-FNRS) of the French-Speaking Community of Belgium.

\section{Authorship contribution}

CF: design of project, collection of the data, drafting of the manuscript and revised it; $\mathrm{CF}, \mathrm{ML}, \mathrm{CH}$ : analysis and interpretation of the data; $\mathrm{ML}, \mathrm{CH}$ : critical revision of the manuscript; $\mathrm{CF}, \mathrm{ML}, \mathrm{CH}$ : Approval of the final version of the manuscript. 


\section{Conflict of interest}

The authors have no conflict of interest with regard to this research.

\section{References}

1. Hummel T, Whitcroft KL, Andrews $P$ et al. Position paper on olfactory dysfunction. Rhinology.2017;54 (supplement 26): 1-30.

2. Doty RL. Olfactory dysfunction in Parkinson disease. Nat Rev Neurol. 2012; 8(6):329-339.

3. McKinnon JH, Demaerschalk BM, Caviness JN, Wellik KE, Adler CH, Wingerchuk DM. Sniffing out Parkinson disease: can olfactory testing differentiate parkinsonian disorders? Neurologist. 2007 Nov;13(6):382-5.

4. Frasnelli J, Schuster B, Hummel T. Interactions between olfaction and the trigeminal system: what can be learned from olfactory loss. Cereb Cortex. 2007; 17(10):2268-2275.

5. Brand G. Olfactory/trigeminal interactions in nasal chemoreception. Neurosci Biobehav Rev. 2006;30(7):908-917.

6. Foguem C, Brand G. Comparison of olfactory thresholds between elderly with Parkinson disease and controls. J Aging Gerontol. 2014;2: 5-12.

7. Thomas P, Hazif-Thomas C. Dépression, présentation clinique et diagnostic chez la personne âgée. Revue Geriatr. 2003; 28: 247-258.

8. Folstein MF, Folstein SE, McHugh PR. "Minimental state". A practical method for grading the cognitive state of patients for the clinician. J Psychiatr Res. 1975;12(3): 189198.

9. Shulman KI. Clock-drawing: is it the ideal cognitive screening test? Int J Geriatr Psychiatry. 2000 Jun;15(6):548-61.

10. Derouesne C, Poitreneau J, Hugonot L, Kalafat M, Dubois B, Laurent B. Mini-Mental State Examination: a useful method for the evaluation of the cognitive status of patients by the clinician. Consensua French version. Presse Med. 1999 Jun 12;28(21):1141-8

11. Hughes AJ, Daniel SE, Kilford L, Lees AJ. Accuracy of clinical diagnosis of idiopathic Parkinson's disease: a clinicopathological study of 100 cases. J Neurol Neurosurg Psychiatry. 1992; 55: 181-184.

12. Foguem C, Novella JL. Maladie de Parkinson du sujet âgé. Revue 'Tout Prévoir -Espace FMC ; dec 2011- jan 2012 ; 427 :27-31.
13. Diederich NJ, Moore CG, Leurgans SE, Chmura TA, Goetz CG. Parkinson disease with old-age onset: a comparative study with subjects with middle-age onset. Arch Neurol. 2003; 60 : 529-33.

14. Belin J, Houéto JL, Constans T, Hommet C de Toffol B, Mondon K. Geriatric particularities of Parkinson's disease: Clinical and therapeutic aspects. Rev Neurol (Paris). 2015; 171(12):841-852.

15. Parashos SA, Luo S, Biglan KM, et al. NET-PD Investigators.Measuring disease progression in early Parkinson disease: the National Institutes of Health Exploratory Trials in Parkinson Disease (NET-PD) experience. JAMA Neurol. 2014 Jun;71(6):710-6.

16. Giladi N, Nicholas AP, Asgharnejad M, et al. Efficacy of Rotigotine at Different Stages of Parkinson's Disease Symptom Severity and Disability: A Post Hoc Analysis According to Baseline Hoehn and Yahr Stage. J Parkinsons Dis. 2016; 6(4): 741-749.

17. Rombaux P, Mouraux A, Bertrand B, Guerit JM, Hummel T. Assessment of olfactory and trigeminal function using chemosensory event-related potentials. Neurophysiol Clin. 2006 Mar-Apr;36(2):53-62.

18. Lasko TA, Bhagwat JG, Zou KH, OhnoMachado L. The use of receiver operating characteristic curves in biomedical informatics. J Biomed Inform. 2005; 38(5):404415.

19. IBM Corp. IBM SPSS Statistics for Windows, Version 19.0. Armonk, NY: IBM Corp. 2010.

20. Hüttenbrink KB, Hummel T, Berg D, Gasser T, Hähner A. Olfactory dysfunction: common in later life and early warning of neurodegenerative disease. Dtsch Arztebl Int. 2013;110(1-2): 1-7.

21. Hummel T, Futschik T, Frasnelli J Hüttenbrink KB. Effects of olfactory function, age, and gender on trigeminally mediated sensations: a study based on the lateralization of chemosensory stimuli.Toxicol Lett. 2003;140-141: 273-280.

22. Suchowersky O, Reich S, Perlmutter J, Zesiewicz T, Gronseth G, Weiner WJ. Quality Standards Subcommittee of the American Academy of Neurology. Practice Parameter: diagnosis and prognosis of new onset
Parkinson disease (an evidence-based review): report of the Quality Standards Subcommittee of the American Academy of Neurology. Neurology. 2006; 66(7): 968975.

23. Schriever VA, Daiber P, Frings $S$, Hummel T. Olfactory-trigeminal interaction in chemosensory perception. Jahrestagung der AG Olfaktologie - Gustologie 2013 - Vorträge. https://www.uniklinikum-dresden.de/.../ Vortraege_Basel_2013.pdf

24. Stern MB, Doty RL, Dotti M, et al. Olfactory function in Parkinson's disease subtypes. Neurology. 1994; 44(2):266-268.

25. Doty RL, Stern MB, Pfeiffer C, Gollomp SM, Hurtig HI. Bilateral olfactory dysfunction in early stage treated and untreated idiopathic Parkinson's disease. J Neurol Neurosurg Psychiatry. 1992; 55(2):138-142.

26. Meusel T, Westermann B, Fuhr P, Hummel $T$, Welge-Lüssen $A$. The course of olfactory deficits in patients with Parkinson's disease-a study based on psychophysical and electrophysiological measures. Neurosci Lett. 2010 Dec 17:486(3):166-70.

27. Ansari KA, Johnson A. Olfactory dysfunction in patients with Parkinson's disease. J Chronic Dis. 1975;28:493-7.

28. Nakayasu C, Kanemura F, Hirano Y, Shimizu $Y$, Tonosaki K. Sensitivity of the olfactory sense declines with the aging in senescence-accelerated mouse (SAM-P1). Physiol Behav. 2000; 70(1-2):135-139.

Dr Clovis Foguem MD, Msc, PhD Auban Moët Hospital

137 rue de l'hôpital

BP: 137

51200 Epernay

France

Tel: 00(33)-6-2112 0819

Fax: 00(33)-3-2658 7171

E-mail:cfoguem@yahoo.fr 